\title{
CONFLICTO Y CONVIVENCIA EN EL DISCURSO PEDAGÓGICO OFICIAL DURANTE EL TARDOFRANQUISMO Y LA TRANSICIÓN DEMOCRÁTICA ESPAÑOLA, 1965-1982
}

Por Cecilia Milito Baraone. Madrid: UNED, 2021, 434 páginas, 978-84-362-7617-6

La modernidad, como dijo en su día Zygmunt Bauman, vino a disolver sólidos, las estructuras sociales, culturales y políticas del Antiguo Régimen, para construir otros sólidos mejores. Los ilustrados del siglo XVIII comprendieron que esas estructuras no eran necesarias, no eran una creación divina, ni siquiera una construcción natural, sino una contingencia, una arbitrariedad que puede alterarse. Muchos científicos sociales han corroborado después esa idea: para Peter L. Berger y Thomas Luckmann, las instituciones, aunque se experimenten como una realidad objetiva, inmutable, tienen una historia y pueden ser modificadas por los individuos; para Marcel Mauss o Pierre Bourdieu, los habitus, los gestos, las formas corporales que sirven habitualmente a la distinción social, no son algo natural, sino aprendizajes que tienden a naturalizarse; lingüistas como Victor Klemperer comprendieron hace mucho tiempo que las prácticas lingüísticas tienden a naturalizar las distintas formas de ver el mundo.

Sin estas premisas, es imposible entender el libro de Cecilia Milito, Conflicto y convivencia en el discurso pedagógico oficial durante el tardofranquismo y la transición democrática española, 1965-1982, publicado dentro de la serie Proyecto MANES que edita la UNED. Su autora se propone escudriñar qué conceptos de "conflicto» y «convivencia» (escolar, social, internacional) trasmiten los discursos educativos oficiales (currículo prescrito, revistas educativas, manuales escolares) en España entre 1965 y 1982, es decir, en el tardofranquismo y la Transición. También Milito es consciente de que las prácticas sociales, los discursos, al institucionalizarse, «se 
convierten en algo natural, dado, normalizado, estabilizado, y dejan de sobresalir; entonces las personas ya no les prestan atención y dejan de preguntarse sobre ellas» (p. 97). Esa naturalización, la simulación de la inevitabilidad de las realidades sociales o políticas, es uno de los procedimientos que permiten a la escuela comportarse como un instrumento de control social. Lo que hace el libro que reseñamos es precisamente descodificar, desnaturalizar esos discursos, comprobar su arbitrariedad, su intencionalidad y potencialidad legitimadora.

Para emprender tal tarea es necesario, por una parte, una buena arquitectura conceptual, y, por otra, una metodología adecuada para descodificar ese discurso que creíamos tan natural, tan de sentido común. El libro de Milito cumple con creces esas dos condiciones. Al primer objetivo dedica el Capítulo 1, donde encontramos una definición detallada de conceptos como organicismo social, funcionalismo, doctrina social de la Iglesia, bien común o teorías del conflicto social, imprescindibles para entender las fuentes que maneja. Y el Capítulo 2, donde se nos aclaran categorías como convivencia (social o escolar) y conflicto (social o escolar). La metodología que va a permitir descodificar, desnaturalizar el discurso sólo podría tener una naturaleza lingüística. Se trata del ACD, el análisis crítico del discurso, del que habló en primer lugar el lingüista Norman Fairclough, y al que se han sumado los seguidores de eso que ha venido en llamarse Lingüística Crítica, que no se conforma con un análisis formal del lenguaje, sino que estudia los procesos y estructuras sociales que esconden los textos: Teun A. van Dijk, profesor de la Universidad Pompeu Fabra de Barcelona, es uno de los más significativos. Efectivamente, Cecilia Milito en el Capítulo 3 de su libro da cuenta del significado social de los procedimientos y trampas que usa el lenguaje hablado o escrito: lexicalizaciones (el uso de unas determinadas palabras implica una decisión ideológica), polarización (el nosotros/ ellos, que tanto refuerza las identidades), eufemismos, figuras de pensamiento... Pero también dedica unas páginas al lenguaje iconográfico que le acompaña en los manuales, profusamente desde los años 70 del pasado siglo.

Cecilia Milito es muy consciente también de la relación bidireccional que hay entre la escuela y el entorno social y político en que ésta se desenvuelve. Por eso, después de describir en el Capítulo 4 las fuentes que utiliza en su libro, tanto primarias (prescriptivas del currículo, 
científico-pedagógicas, didácticas, manuales) como secundarias (bibliografía), dedica el Capítulo 5 a analizar el contexto histórico en que esas fuentes aparecen, el tardofranquismo (1959-1975) y la transición a la democracia (1976-1982). El contexto educativo correspondiente es analizado en el Capítulo 6, que aborda la transición de un sistema educativo elitista a uno tecnocrático de masas, y en el Capítulo 7, que analiza los cambios curriculares que le acompañan: Cuestionarios Nacionales para la Enseñanza Primaria de 1965, Ley General de Educación (LGE) de 1970 y Nuevas Orientaciones Pedagógicas para la Educación General Básica (EGB), prestando especial atención a las materias o unidades didácticas donde se reflejan los conceptos de conflicto y convivencia en que se centra este trabajo: Área Social, Educación Cívica (desde 1974, Educación Cívico- Social).

Los tres últimos capítulos constituyen realmente el cuerpo del libro, pues estudian por orden cronológico cómo representan los documentos oficiales esos conceptos. El Capítulo 8 estudia el tardofranquismo hasta la aprobación de la LGE, es decir, el quinquenio que va de 1965 a 1970. Milito comprueba que las fuentes primarias (Cuestionarios de 1965, la revista Vida Escolar, los manuales) insisten ante todo en la disciplina y el orden, y, con la doctrina social de la Iglesia como referente, entienden la convivencia escolar como armonía y disuasión del conflicto. El concepto de convivencia social, que se inspira en la misma fuente, obedece a un patrón organicista y funcionalista, mientras la convivencia internacional se aborda desde un punto de vista muy ingenuo: se habla de colaboración, entendimiento entre los países... El resultado es el disimulo, el olvido de los conflictos sociales o internacionales y de las causas que los motivan. Es la visión armoniosa de la sociedad, de la vida política y de la vida internacional que quiere ofrecer la dictadura de Franco.

El Capítulo 9 aborda el tardofranquismo después de la aprobación de la LGE, es decir, el quinquenio 1970-1975. Cecilia Milito observa algunos cambios respecto al periodo anterior: aunque con contradicciones, aprobada la LGE, se habla cada vez más de cooperación y trabajo en equipo para formular la convivencia escolar, se habla puntualmente de participación en la toma de decisiones e incluso, aunque con eufemismos, puede aceptarse la existencia del conflicto social (la denominada cuestión social que plantea la Revolución Industrial), o de la Guerra Fría... Pero, todavía vigente la dictadura, dominan las continuidades: 
concepciones organicistas y armoniosas de la vida social inspiradas en la doctrina social de la Iglesia, visión ingenua de las relaciones internacionales, ocultación del conflicto y de las contradicciones sociales, lo que llama especialmente la atención, dada la conflictividad social y política o incluso la violencia que en esos momentos vive España en sus calles.

El esquema continuidades/cambios sirve también a la autora del libro para analizar en el Capítulo 10 el periodo de la Transición (19761982). Tímidamente las fuentes primarias introducen valores democráticos para explicar la convivencia escolar, pero sin abandonar del todo el discurso sobre la armonía, la concordia y la colaboración propio de un pasado autoritario, mientras los conflictos escolares sólo se tratan con eufemismos. No se ahonda suficientemente en los conflictos internacionales. La convivencia social se plantea muchas veces en términos organicistas y funcionalistas muy idealizados (armonía, paz, bien común) de clara raigambre religiosa, mientras los conflictos sociales y políticos, especialmente si tienen que ver con el pasado más reciente de España (Guerra Civil, franquismo), siguen siendo tabú. El silencio de la escuela, en definitiva, sigue contrastando con el ruido que provocan los conflictos que estallan en la calle durante la Transición: la autora deja clara la relación entre ese silencio y el tipo de transición a la democracia que se produce en España. En las conclusiones, entre otras cosas, Milito hace una interesante valoración sobre el peso que ha tenido el organicismo para explicar la convivencia y los conflictos escolares y sociales que, paradójicamente, no pierde la perspectiva individualista. Individualismo que (y aquí recurre a Noam Chomsky) sirve para atomizar a los individuos, para sustraerles su capacidad de organizarse y de cambiar el mundo.

Sin duda, el tema de la convivencia y de la conflictividad en la escuela, en la sociedad, en las relaciones internacionales ofrece muchas más posibilidades. Se me ocurre ampliar el campo de observación, más allá de la Educación Primaria y de la EGB, al currículo de Bachillerato y, más allá de la asignatura de Educación Cívica (o similar), a la asignatura de Historia y Ciencias Sociales. O escudriñar, además de la encíclica Pacem in Terris, ciertas fuentes eclesiales como los documentos del Vaticano II, especialmente la Constitución Gaudium et Spes (participación política, dignidad humana, rechazo a los sistemas tiránicos), que tienen un reflejo directo en las fuentes que trabaja la autora. Otro tanto cabría decir de la versión particular que ofrece el partido único del régimen, 
FET y de las JONS, del organicismo y del funcionalismo: la evolución interna del Movimiento en el tardofranquismo es un asunto muy poco conocido. Habría que prestar atención también al traslado casi directo del discurso político del momento a los documentos escolares: ocurre, por ejemplo, con el discurso aperturista de Carlos Arias Navarro a la altura de 1973, que habla de participación política y «contraste de pareceres» a través de las asociaciones (que no partidos políticos). Sin duda, abordar el tema de la convivencia y el conflicto en otros periodos permitirá perspectivas más amplias y enriquecedoras: sería interesante, por ejemplo, examinar el discurso del primer franquismo, el de la Iglesia integrista previa al Concilio Vaticano II y el discurso del partido único antes de ser contaminado por valores tecnocráticos y modernizantes.

Cecilia Milito marcó un camino, pero no va a poder andarlo más. La vida, siempre aliada con la muerte, está ya muy acostumbrada a dar empujones brutales. Nos queda el mensaje de fondo que trasmite su libro, que, por un lado, nos alerta sobre los engaños, las manipulaciones que muy frecuentemente acompañan al sistema educativo, sobre todo cuando nos quiere convencer de que la realidad y el presente son algo natural y necesario. Y, por otra, sin dejarse contaminar por el escepticismo de raigambre tradicional o posmoderna, apela a la necesidad de desnaturalizar la realidad, de disolver sólidos para construir otros mejores. Cecilia fue muy consciente de que para conseguir tal cosa no había que ocultar el conflicto, sino entenderlo como un valor, como una oportunidad. Y de que la escuela no puede seguir dando la espalda a la realidad, al conflicto, a la vida. Sit terra tibi levis.

Emilio Castillejo Cambra. Centro Asociado de la UNED. Pamplona ecastillejo@Pamplona.uned.es 